\section{Hypertonic Saline: Safe therapy for Children with Acute Brain Insult in Emergency Department of Low and Middle Income Country}

\section{Abstract}

Hypertonic saline (HTS) has been used for some years to treat elevated intracranial pressure in children in high income countries. There is limited safety data from low and middle income countries.

Objective: The primary objective of this study was to assess the immediate response and safety of intravenous administration of hypertonic saline in children with acute brain injury presenting to the pediatric emergency department (PED) of Aga Khan University Hospital (AKUH). The secondary outcomes were changes in outcome- predictive physiological parameters.

Methods: A retrospective, chart review of children who received intravenous HTS in the pediatric emergency department of AKU between January 2013 and December 2013 to treat acute brain injury (ABI). We studied both changes in physiological parameters and adverse effects related to HTS therapy.

Results: In the period studied, 216 children received intravenous bolus of $3 \%$ HTS as part of their initial management in the PED. The median age of the patients was 6.1 years ( 1 month to 16 years) and a median dose of $5 \mathrm{~mL} / \mathrm{kg}$ with $98 \%$ of doses given via peripheral line between 30 minutes to one hour. Diagnosis included traumatic brain injury in 110 patients (50.9\%) and non-traumatic injury 106 (49.1\%) including CNS infection (43.4\%), intracranial bleeding (7.5\%), acute hepatic failure (10.4\%), diabetic ketoacidosis with altered mental status (4.7\%). Clinical indications included: depressed consciousness (75.5\%), seizures (24\%) and shock (0.5\%). Significant improvement was observed in both heart rate ( $p$ value < 0.05 ) and GCS ( $p$ value $=0.001$ ) after $3 \%$ HTS therapy No adverse events related to the HTS or its route of administration were noted in any child.

Conclusion: We found 3\% HTS was safe in children with acute brain injury traumatic or non-traumatic brain injury.

Keywords: Hypertonic saline; Traumatic brain injury; Cerebral edema; Depressed consciousness

\author{
Nadia Mohammad ${ }^{1}$, \\ Surraiya Banu², \\ Nick Brown ${ }^{3}$, \\ Sidra Kaleem ${ }^{4}$, \\ Saleem Akhtar ${ }^{5}$ and \\ Anwar-ul-Haq ${ }^{6}$
}

1 Aga Khan University Hospital, Karachi, Pakistan.

2 Aga Khan University Hospital, Karachi, Pakistan.

3 Department of child health, AKU, Salisbury foundation trust hospital, Karachi, Pakistan.

4 Aga Khan University Hospital, Karachi, Pakistan.

5 Aga Khan University Hospital, Karachi, Pakistan.

6 Aga Khan University Hospital, Karachi, Pakistan.

Corresponding author:

Nadia Mohammad

Đ nadia.mohammad@aku.edu

Aga Khan University Hospital, Karachi, Pakistan.

Tel: 00923243596131

Citation: Mohammad N. Hypertonic Saline: Safe therapy for Children with Acute Brain Insult in Emergency Department of Low and Middle Income Country. J Pediatr Care. 2017, 3:1.

Received: December 06, 2016; Accepted: January 20, 2017 ; Published: January 25, 2017

\section{Introduction}

Acute brain injury $(A B I)$ has a diverse etiology from traumatic to non- traumatic (ischemic stroke, intraparenchymal or subarachnoid hemorrhage, infection, or toxic-metabolic derangements [1]. Traumatic brain injury (TBI) is an important global public health problem. Globally at least 10 million TBIs are serious enough to result in death or hospitalization annually [2]. Mortality in children with severe TBI is often the result of a refractory increase in intracranial pressure (ICP) [3]. Raised ICP causes decreased cerebral perfusion pressure (CPP) and if left untreated will lead to poor outcome $[3,4]$. Improving CPP 
is the cornerstone of management and osmotic therapy is one modality of treatment commonly used in high income countries (HICs) adjunctively with other general measures (head position, hyperventilation).

Management of raised ICP should be aimed at preventing secondary brain injury such as hypoxemia, hypercapnia, hyponatremia, hyperthermia, seizures and hypo/hyperglycemia and increase in ICP. First-tier Therapies for Raised ICP [5] include head elevation at $30^{\circ}$ can improve venous drainage and lower ICP without adversely affecting MAP or CPP, osmotic therapy, sedation and analgesia and hyperventilation [3]. Intravenous Hypertonic saline (IHS) treatment is attractive as it is simple, noninvasive, and safe therapeutic osmotic agent $[6,7]$.

Hypertonic saline (HTS) has immunomodulatory and antiinflammatory properties; it reduces cerebral edema through an osmotic gradient between the intravascular and intracellular/ interstitial compartments and improves cerebral perfusion pressure by improving plasma volume expansion [8]. The standard dose of HTS is $3-5 \mathrm{ml} / \mathrm{kg}$. There are several reports from High Income Countries (HICs) demonstrating efficacy and safety of HTS in critically ill children $[7,9,10]$.

There is, however, very limited data from pediatric emergency room on the use of HTS from low and middle income countries (LMICs) like Pakistan where acute neurological disorder has been estimated to constitutes about $19.3 \%$ of PICU admission [11]. The purpose of this study was to assess the response and safety of HTS in Acute Brain Injury in the acute care setting of Pediatric emergency Unit.

\section{Methodology}

This was a retrospective, case note study undertaken at the PED of Aga Khan University Hospital (AKUH). AKUH is one of the largest private sector tertiary care hospitals in Pakistan with advanced and well equipped 10 bedded pediatric ER run by pediatric emergency team included faculty, supervisor, residents and paramedical staff, with a volume of approximately 16000 patients annually. Study approval was obtained from the Institutional Ethical Review Committee of AKUH (ERC \# 3122). Hypertonic saline (3\%) has been standard osmotic therapy for correction of symptomatic hyponatremia for the last 10 years in our PED and PICU. We retrieved the medical record number of patients who visited the emergency department and identified patients in whom 3\% HTS was used through the AKU pharmacy data base in the 12 months between (January and December 2013).

The demographic and clinical characteristics including age, gender, primary diagnosis, indication for use of hypertonic saline, result of brain imaging done at AKUH and outcome were abstracted from the patient's medical record onto a structured data sheet. Site of dose administration, time duration over which dose was administered and total number of doses administered were recorded from nursing documentation. Indications for IHS were taken from PED physician documentation and crosschecked with final hospital discharge summaries to ensure correct categorization. We did not have routine facility for invasive monitoring of intracranial pressure or Non-invasive transcranial Doppler (TCD) and therefore, used clinical criteria [non-invasive hemodynamics (Heart rate and Blood pressure), Neurological (Glasgow Coma Score (GCS)] [12] and change in serum sodium in order to indirectly measure response. Blood pressure was measured by non-invasive oscillometric technique and GCS was observed by trained doctor, and categorized in mild, moderate and severe according to GCS score. Vitals signs were recorded before and 1 hour after HTS infusion through one hourly nursing sheet documentation. Serum sodium values were measured in laboratory by ion selective electrode method from auto analyzer. Serum sodium was taken before HTS infusion and 6 hours after HTS infusion. Hyponatremia was defined as a serum sodium level of $<135 \mathrm{meq} / \mathrm{L}$ and hypernatremia as serum sodium level $>155 \mathrm{meq} / \mathrm{L}$. All records pertaining to the patient's PED visit, nursing documentation throughout hospitalization, and discharge or death summaries were also explored for evidence of known adverse effects of IHS administration including renal failure, hypokalemia hyperkalemia, hypernatremia and phlebitis at the site of infusion.

Acute kidney injury (AKI) is diagnosed based on pediatric RIFLE criteria (Risk, Injury, Failure, Loss of kidney function, and End stage kidney disease), criteria based on changes in serum creatinine from baseline levels [13]. Hypokalemia is defined as serum potassium level $<3.5 \mathrm{meq} / \mathrm{L}$ while hyperkalemia as serum potassium level $>5.5 \mathrm{meq} / \mathrm{L}$.

It is our standard care of nursing when high osmolar fluid transfused through peripheral intravenous line assessment of the cannula site done hourly to check for phlebitis. All Computed Tomography (CT) scan of brain was done as part of initial evaluation in the PED. All CT was read by pediatric radiologist and they were blinded to patient's diagnosis.

Data analysis was undertaken by Statistical Package for Social Science (SPSS) software, Version 19. Data was presented as mean \pm SD for continuous, normally distributed variables such as age, weight of the patient, vitals and serum sodium before and after administration of HTS. Frequency and percentages was computed for qualitative variables like gender, diagnosis of patient, indication of HTS, CT scan findings and GCS categories [mild $(>12)$, moderate $(8-12)$, severe $(<8)$ ]. Student $t$ test was used for comparing continuous variable, Pearson chi square test was used for comparing categorical variables and $p<0.05$ was considered as significant.

\section{Results}

Of total 15,723 children visited to the PED during study period, $216(1.4 \%)$ received intravenous bolus of $3 \%$ of HTS in PED. Mean age of the patients was 6.1 years (0-16years) and median age was 5 year. Out of 216 children 146 (67.6\%) were male. Table 1 shows the patient's characteristics of clinico-demographic variables.

The majority of patients $(72.7 \%)$ received HTS in dose of $5 \mathrm{ml} /$ $\mathrm{kg}$ which was infused over 30 minutes to 1 hour. 212 (98\%) of patients received HTS via peripheral line. 
Table 1: Clinico-demographic characteristics $(\mathrm{N}=216)$.

\begin{tabular}{|c|c|}
\hline Demographic characteristics & Number (\%) \\
\hline Age in yrs (Mean/SD) & $6.1(+/-4.36)$ \\
\hline$<1$ yrs & $24(11.1 \%)$ \\
\hline$>1$ yrs & $192(88.8 \%)$ \\
\hline Weight (kg) (Mean/SD) & $20.2(+/-13.04)$ \\
\hline \multicolumn{2}{|c|}{ Gender } \\
\hline Male & $146(67.6 \%)$ \\
\hline Female & $70(32.4 \%)$ \\
\hline Clinical characteristics & Number (\%) \\
\hline \multicolumn{2}{|c|}{ Diagnoses } \\
\hline TBI & $110(50.9 \%)$ \\
\hline Non-TBI & $106(49.1 \%)$ \\
\hline Primary CNS infection & $46(43.4 \%)$ \\
\hline Acute hepatic failure & $11(10.4 \%)$ \\
\hline Intracranial bleeding & $8(7.5 \%)$ \\
\hline Brain tumor & $8(7.5 \%)$ \\
\hline Seizure disorder & $7(6.6 \%)$ \\
\hline Secondary infection & $8(7.5 \%)$ \\
\hline DKA & $5(4.7 \%)$ \\
\hline Other & $13(12.3 \%)$ \\
\hline \multicolumn{2}{|c|}{ Indications } \\
\hline Depressed consciousness & $163(75.5 \%)$ \\
\hline Seizures & $52(24 \%)$ \\
\hline Shock & $1(0.5 \%)$ \\
\hline Radiology done & 151 (69.9\%) \\
\hline CT (Normal) & $56(29.1 \%)$ \\
\hline CT (Abnormal) & $95 / 151(62.9 \%)$ \\
\hline Edema & $39 / 95(41 \%)$ \\
\hline Midline shift & $3 / 95(3.1 \%)$ \\
\hline $\mathrm{ICH}$ & $53 / 95(55 \%)$ \\
\hline \multicolumn{2}{|c|}{ Outcomes } \\
\hline Alive & $183(84.7 \%)$ \\
\hline Expired & $33(15.3 \%)$ \\
\hline \multicolumn{2}{|c|}{$\begin{array}{l}\text { Note: TBI: Traumatic Brain Injury, ICH: Intracranial Hemorrhage, LAMA: } \\
\text { Leave Against Medical Advice, DKA Diabetic Ketoacidosis, Other: Stroke, } \\
\text { Congenital Hydrocephalus, Sub Acute Sclerosing Panencephalitis (SSPE), } \\
\text { Hypoxic Ischemic Encephalopathy (HIE), Congenital Hydronephrosis, } \\
\text { and Drug Overdose. }\end{array}$} \\
\hline
\end{tabular}

Diagnosis for IHS included TBI in 110 patients (50.9\%) and nontraumatic injury (NTBI) 106 (49.1\%). CNS infection was the most common etiology of NTBI (43\%) followed by acute hepatic failure (10.4\%), other causes included stroke, congenital hydrocephalus, sub-acute sclerosing panencephalitis (SSPE), hypoxic ischemic encephalopathy (HIE), congenital hydronephrosis, and drug overdose (Table 1). The most common clinical indication for IHS was depressed level of consciousness 163 (75.5\%) followed by seizure 52 (24\%). Out of 52 patients with seizure; 44 (85\%) had hyponatremia and $8(15 \%)$ had seizure without hyponatremia.

Axial brain imaging [Computed Tomography (CT)] was performed in 87 (79\%) TBI patients and 64 (60\%) NTBI patients, Abnormal finding was observed in 51 (58.6\%) and 44 (68.7\%) of TBI and NTBI patients respectively. In TBI patients ICH was found in 42 (82.4\%), cerebral edema in $8(15.7 \%)$ and mid line shift in $1(2 \%)$ patients, whereas cerebral edema was most common finding seen in 31 (47\%) of NTBI patients followed by ICH in 11 (16.4\%) and Midline shift in 2 (2.9\%) respectively. 101 (46.8\%) patients were admitted in pediatric intensive care unit (PICU), 60 (27.8\%) in special care unit (SCU), 21 (9.7\%) in ward, 19 (8.8\%) were discharged from ER in stable condition and 15 (6.9\%) were Leave against medical advice (LAMA) from ER. Out of 216 patients 33 (15.3\%) of patients were died, because of severity of primary illness.

We observed improvements in metabolic and clinical profile after $3 \%$ HTS therapy, level of serum sodium was changed from $133 \mathrm{mmol} / \mathrm{L}$ to $138 \mathrm{mmol} / \mathrm{L}(p$-value $<0.000)$ and heart rate was changed from $120 \mathrm{~b} /$ minute to $116 \mathrm{~b} /$ minute ( $p$-value of $<0.000$ ) (Table 2). After HTS infusion there was change in GCS, $10 \%$ of children with severe group of GCS improved to moderate group of GCS and $12 \%$ of children improved from moderate to mild group of GCS ( $p$ value $<0.001$ ). No adverse effects were noted in our study group.

\section{Discussion}

We found that use of HTS is safe in children with ABI in PED in low middle income country. Management of raised ICP in the "Initial Platinum Minutes" is critical to improving outcomes. Osmotic therapy (mannitol and hypertonic saline) is routinely used in a wide range of acute conditions including head injury, ischemic stroke and intracerebral hemorrhage [14]. Over the last few years, the use of HTS has become established practice for suspected raised ICP in pediatric TBI patients in HICs [3]. In the 2012 pediatric TBI guidelines, there was greater evidence of effect for HTS than for mannitol [15]. Several studies have shown equipoise in the efficacy of mannitol in reducing mortality in head injury [16] and others found HTS was superior [17].

Patients with TBI represent the largest group to receive HTS in our study. Although ICP measurements were not obtained and comment cannot be made on effectiveness or impact on outcome, $3 \%$ saline appears to be safe to use in these patients in the acute setting.

The literature from HICs (both adult and pediatric) has shown low rates of complications in a PICU setting $[7,9,18,19]$. Our study demonstrates that administration of $3 \%$ HTS through peripheral IV line is safe. In, another pediatric study, Luu et al. retrospectively reviewed the use of $3 \%$ HTS during critical transports over a 4 year period [20]. In that study, 101 children received $3 \%$ HTS during transport to the hospital; $>90 \%$ were treated for suspected cerebral edema or intracranial bleeds. Notably, in $96 \%$ of cases the initial infusions were administered through peripheral intravenous lines. No local reactions, renal abnormalities, or central pontine myelinolysis were observed.

\section{Conclusion}

HTS has been found to be safe and effective in the treatment of hyponatremic seizures in children [21], in our cohort forty four children with hyponatremic seizures received IHS as bolus with resolution of seizure in each case, similar response was shown by Timothy et al. [9]. In our cohort, ED physicians used 
Table 2: Comparison of clinical and metabolic profile before and after HTS Intervention.

\begin{tabular}{|c|c|c|c|}
\hline Clinical profile & Baseline & After HTS administration & P-value \\
\hline $\mathrm{H} / \mathrm{R}(\mathrm{b} / \mathrm{min})(\mathrm{mean} / \mathrm{SD})$ & $120.9(+/-27.09)$ & $116.5(+/-25.42)$ & 0.000 \\
\hline $\begin{array}{l}\text { BP systolic }(\mathrm{mmHg}) \text { (mean/ } \\
\text { SD) }\end{array}$ & $102.4(+/-17.78)$ & $103.8(+/-15.30)$ & 0.064 \\
\hline \multicolumn{4}{|c|}{ GCS Score } \\
\hline Mild (>12) & $122(56.5)$ & $128(59.3)$ & \multirow{3}{*}{0.001} \\
\hline Moderate (8-12) & \multirow{2}{*}{$\begin{array}{c}54(25) \\
40(18.5)\end{array}$} & $51(23.6)$ & \\
\hline Severe $(<8)$ & & $37(17.1)$ & \\
\hline \multicolumn{4}{|c|}{ Metabolic profile } \\
\hline \multicolumn{2}{|c|}{ Serum sodium (mmol/L) (mean/SD) } & $138.5(+/-7.78)$ & 0.000 \\
\hline
\end{tabular}

IHS in all those patients who presents to ED with depressed level of consciousness in previously healthy acutely ill children with highly suspicious of hyponatremic encephalopathy where incidence of hyponatremia was about $30 \%$ (unpublished data). Sunil et al. reported hyponatremia in $29.8 \%$ in sick children seeking pediatric emergency care [22].

Strength of our study includes novelty. It is the first publication from a developing ED to assess safety of HTS. Limitations were that it was single centre and retrospective design, without a control group, we were also unable to undertake continuous ICP monitoring. In sedated and intubated patient we were unable to assess the GCS and we did not use age-appropriate norm for vitals, we used baseline vitals to assess the effect of therapy.

The use of HTS solution is safe in children with $A B I$ in a carefully managed ED in an LMIC and may improve outcome from a wide range of acute brain injuries in children. 


\section{References}

1 Bhardwaj A, Ulatowski J (2004) Hypertonic saline solutions in brain injury. Curr Opin Crit Care 10: 126-131.

2 Langlois JA, Brown RW, Wald M (2006) The epidemiology and impact of traumatic brain injury: A Brief Overview. J Head Trauma Rehabil. 2: 375-378.

3 Kukreti V, Mohseni-Bod H, Drake J (2014) Management of raised intracranial pressure in children with traumatic brain injury. J Pediatr Neurosci. 9: 207-215.

4 Castillo LR, Shankar Gopinath S, Robertson CS (2008) Management of intracranial hypertension. Neurol Clin. 26: 521-541.

5 Adelson PD, Bratton SL, Carney NA (2003) Guidelines for the acute medical management of severe traumatic brain injury in infants, children, and adolescents. Chapter 17. Critical pathway for the treatment of established intracranial hypertension in pediatric traumatic brain injury. Pediatr Crit Care Med 4: 65-67.

6 Larive LL, Rhoney DH, Parker D (2004) Introducing hypertonic saline for cerebral edema: An academic center experience. Neurocrit. Care. 1: $435-440$.

7 Peterson B, Khanna S, Fisher B (2000) Prolonged hypernatremia controls elevated intracranial pressure in head-injured pediatric patients. Crit. Care Med. 28: 1136-1143.

8 Forsyth L, DeRyke X, Parker D (2008) Role of hypertonic saline for the management of intracranial hypertension after stroke and traumatic brain injury. Pharmacotherapy 28: 469-484.

9 Timothy E, Cristina M, Sheila P (2013) Intravenous hypertonic saline use in the pediatric emergency department. Pediatric Emergency Care 29: 71-73.

10 Khanna S, Davis D, Peterson B, et al. (2000) Use of hypertonic saline in the treatment of severe refractory posttraumatic intracranial hypertension in pediatric traumatic brain injury. Crit Care Med. 28: 1144-1151.
11 Abbas Q, Amber Shabbir A, Siddiqui N (2014) Burden of neurological illnesses in a pediatric intensive care unit of developing country. Pak J Med Sci 30: 1223-1227

12 Teasdale G, Jennett B (1974) Assessment of coma and impaired consciousness. A practical scale. Lancet 2: 81-84.

13 Gonda D (2013) Complications associated with prolonged hypertonic saline therapy in children with elevated intracranial pressure. Neurocritical Care 14: 611.

14 Diringer MN (2013) New trends in hyperosmolar therapy. Curr Opin Crit Care. 9: 77-82.

15 Kochanek PM, Carney N, Adelson PD (2012) Guidelines for the acute medical management of severe traumatic brain injury in infants, children, and adolescents - Second edition. Pediatr Crit Care Med 13: 1-82.

16 Walcott BP, Kahlen KT, Simard JM (2012) Novel treatment targets for cerebral edema. Neurotherapeutics 9: 65-72.

17 Cruz J, Minoja G, Okuchi K (2004) Successful use of the new highdose mannitol treatment in patients with Glasgow Coma Scale scores of 3 and bilateral abnormal pupillary widening: a randomized trial. J Neurosurg 100: 376-383.

18 Froelich M, Ni Q, Wess C (2009) Continuous hypertonic saline therapy and the occurrence of complications in neurocritically ill patients. Crit Care Med 37: 1433-1441.

19 White H, David Cook D, Venkatesh B (2006) The use of hypertonic saline for treating intracranial hypertension after traumatic brain injury. Anesth Analg 102: 1836-1846.

20 Luu JL (2011) Three-percent saline administration during pediatric critical care transport. Pediatr Emerg Care. 27: 1113-1117.

21 Sarnaik AP, Meert K, Hackbarth R (1991) Management of hyponatremic seizures in children with hypertonic saline: A safe and effective strategy. Crit Care Med 19: 758-762.

22 Prasad SV, Singhi S, Chugh KS (1994) Hyponatremia in sick children seeking pediatric emergency care. Indian Pediatr. 31: 287-294. 